\title{
Childblain Lupus Erythematosus - Clinical Case and Review
}

\author{
Cernadas E*, AC Dionisio, AP Gomes, Corrêa J, Estevão D, Vicente L
}

Centro Hospitalar Universitário Cova da Beira, Covilha, Portugal

DOI: $10.36347 /$ sjmcr.2020.v08i08.006

| Received: 03.04.2020 | Accepted: 11.04.2020 | Published: 13.08.2020

*Corresponding author: Cernadas E

\section{Abstract}

Chilblain Lupus Erythematosus is a rare form of cutaneous lupus erythematosus (CLE) more frequently seen in middle aged females. It is characterized by erythematous to violaceous plaques over the acral areas induced by exposure to cold, unlike photosensitivity cutaneous CLE lesion. The authors present a case of chilblain lupus erythematosus in a middle age female, it's clinical diagnosis and therapeutic options. The differential diagnoses of a purpuric skin rash include chilblain lupus erythematosus, idiopathic chilblains and lupus pernio.

Keywords: anti-nuclear antibody, chilblain lupus erythematosus, chronic cutaneous lupus erythematosus. Copyright @ 2020: This is an open-access article distributed under the terms of the Creative Commons Attribution license which permits unrestricted use, distribution, and reproduction in any medium for non-commercial use (NonCommercial, or CC-BY-NC) provided the original author and source are credited.

\section{INTRODUCTION}

Chilblain lupus erythematosus is a rare form of chronic cutaneous lupus erythematosus [1], for which there is not much literature to date. It is characterised by symmetrically distributed circumscribed erythematous to violaceous pruriginous or painful plaques over dorsal and lateral aspects of hands and feet, appearing during cold, damp weather conditions.

\section{CaSe Report}

The authors present the case of a 63-years-old women presented in internal medicine consultation with a 2 months history of an itchy and painful erythematous rash on the fingers of both hands associated with finger's swelling. The patient had no previous history of medical or surgical problems and was not taking any kind of medication. The rash started at the distal portion of the $3^{\text {rd }}$ and $4^{\text {th }}$ fingers of left hand and spread proximally (Picture 1).

She underwent a set of standard blood tests including a full blood count, urea and electrolytes, C reactive protein and clotting screen which were all normal. The erythrocyte sedimentation rate was $36 \mathrm{~mm}^{3} / \mathrm{h}$ and there was a hypergammaglobulinaemia. Immunological screening was positive for ANA, rheumatoid factor and anti-Ro (SS-A)/LA (SS-B) antibodies. Cryoglobulin, cold agglutinin, anti-DNA and c-ANACS studies were negative. Screening for antiphospholipid antibodies was negative. Serologic studies for viral infections were negative, as well as microbiological cultures. She underwent a skin punch biopsy for immunofluorescence, which showed fibrinogen deposition in the dermal vessels; the histology displayed perivascular lymphocyte infiltrates in the dermis, hyperkeratosis and necrotic keratinocytes. These findings were consistent with the diagnosis of chilblain lupus erythematosus. The patient was medicated with hydroxychloroquine $400 \mathrm{mg}$ id, however without response. At the 4th month topical thalidomide was started and controlled on $8^{\text {th }}$ month's consultation. The lesions were greatly improved with only a minor scar on the $4^{\text {th }}$ lateral scar.

\section{DiscuSSION}

Chilblain lupus erythematosus is a rare form of chronic cutaneous lupus erythematosus [2], more frequently seen in young females. Only a few cases have been report, and the first report was dated at 1888 by Hutchinson. It is characterised by purple plaques and oedematous skin mainly around the acral regions of the body, which are most frequently exposed to the cold [3], such as the toes, fingers, ears and nose. The rash is characterised by tender plaques that have a purple discolouration, nodules (which may have central erosions/ulcerations) and oedematous skin. The lesions tend to start off as a pruritic rash and thereafter become tender. The underlying pathophysiology is of an autoimmune nature [4] and most cases of chilblain lupus erythematosus are sporadic triggered by coldinduced microvascular injury or vasoconstriction associated with stasis and hyperviscosity. Childblain Lupus Erythematosus lesions may be clinically indistinguishable from simple Childblain or perniosis of healthy individuals. There have been cases noted of a 
rare familiar form, which is inherited in an autosomal dominant manner. Familiar form of Chilblain lupus manifests in early childhood and is caused by a heterozygous mutation in the TREXI gene that encodes a 3'-5' DNA exonuclease [3, 4]. Histological features are vacuolar interface dermatitis, perivascular lymphocytic infiltration and papillary oedema and dermo epidermal junction deposition of $\operatorname{IgM}, \operatorname{IgA}, \mathrm{C} 3$ and fibrinogen in Immunofluorescence study. Patients with chilblain lupus erythematosus may also display hypergammaglobulinaemia (over 2/3), positive rheumatoid factor (in 50\%), antinuclear antibody, antiphospholipid or anti-Ro antibodies. They are usually negative for antidouble-stranded DNA antibodies. Approximately $20 \%$ of patients go on to develop systemic lupus erythematosus. Su et al., proposed the Mayo Clinic Diagnostic criteria for a confirmative diagnosis of CHLE. Major criteria [5] include skin lesions in acral locations induced by exposure to cold or a drop in temperature, and evidence of lupus erythematosus in the skin lesions by histopathologic examination or DIF. Minor criteria are the coexistence of SLE or other skin lesions of Discoid Lupus Erythematous; response to anti-lupus erythematosus therapy; and negative results of testing for cryoglobulin and cold agglutinins. Diagnosis of CHLE is confirmed if patient fulfills both the major and any one of the minor criteria. The first-line treatment for mild and localised cutaneous lupus erythematosus is topical corticosteroids or antimalarials. Some studies have also shown benefit from the use of topical tacrolimus and pimecrolimus or mycophenolate mofetil.

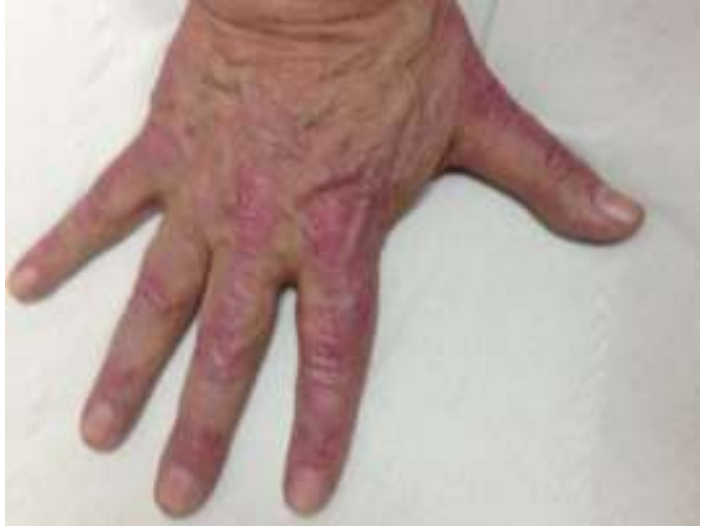

Picture-1

\section{REFERENCES}

1 Hutchinson J. Harveian Lectures on Lupus: The varieties of common lupus. Br Med. J. 1888; 1:5863.

2 Su WP, Perniciaro C, Rogers RS. Chilblain lupus erythematosus (lupus pernio): clinical review of the Mayo Clinic experience and proposal of diagnostic criteria. Cutis. 1994; 54:395-9.

3 AlMahameed A, Pinto DS. Pernio (chilblains). Curr Treat Options Cardiovasc Med. 2008; 10:12835.

4 Gouillon L, Debarbieux S. Chilblain lupus erythematosus treated successfully with mycophenolate mofetil. Int J Dermatol. 2017 Aug; 56(8):e158-e159.

5 Hedrich CM, Fiebig B, Hauck FH. Chilblain lupus erythematosus-a review of literature. Clin Rheumatol. 2008; 27:949-54. 